\title{
Application of the Variational Iteration Method for Inverse Stefan Problem with Neumann's Boundary Condition
}

\author{
Damian Słota \\ Institute of Mathematics \\ Silesian University of Technology \\ Kaszubska 23, 44-100 Gliwice, Poland \\ d.slota@polsl.pl
}

\begin{abstract}
In this paper, the possibility of application of the variational iteration method for solving the inverse Stefan problem with a Neumann boundary condition is presented. This problem consists in a calculation of temperature distribution as well as in the reconstruction of the function which describes the heat flux on the boundary, when the position of the moving interface is known. The validity of the approach is verified by comparing the results obtained with the analytical solution.
\end{abstract}

Keywords: Inverse Stefan problem, Variational iteration method, Heat equation, Solidification.

\section{Introduction}

In this paper, the author is trying to solve the one-phase inverse design Stefan problem with a Neumann boundary condition. This problem consists in a calculation of temperature distribution as well as in the reconstruction of the function which describes the heat flux on the boundary, when the position of the moving interface is known. This paper applies the variational iteration method to the discussed problems. The variational iteration method was developed by Ji-Huan He [1, 2, 3, 4, 5, and is useful for solving a wide range of problems [1, 2, 3, 7, 5, 8, 9, 4, 6, 10, 11. The application of the variational iteration method for direct and inverse Stefan problems with a Dirichlet boundary condition is considered in paper 12 .

It is possible to find an exact analytical solution of the inverse Stefan problem only in few simple cases. In other cases we are left with approximate solutions only [15, 17, 18, 16, 14, 13. For example in papers 14, 13, authors used the Adomian decomposition method combined with optimization for an approximate solution of a one-phase inverse Stefan problem. However, in paper [17, the authors compare selected numerical methods to solve a one-dimensional, one-phase inverse Stefan problem. 


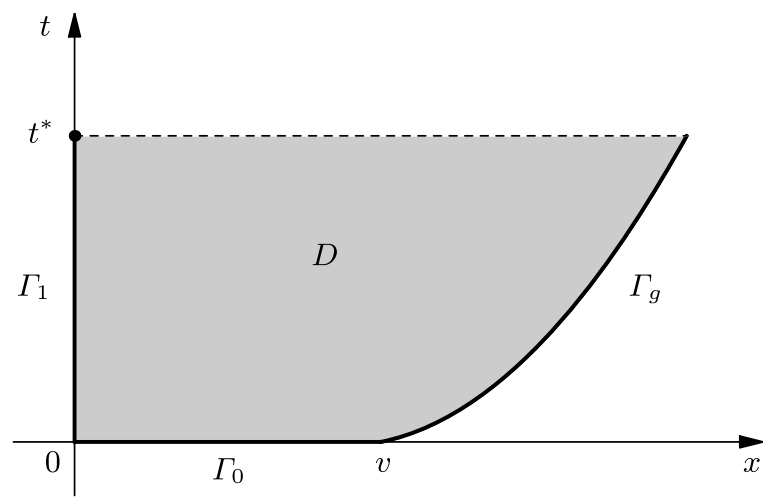

Fig. 1. Domain of the problem

\section{Problem Formulation}

Let $D=\left\{(x, t) ; t \in\left[0, t^{*}\right), x \in[0, \xi(t)]\right\}$ be a domain in $\mathbb{R}^{2}$ (Figure 1). On the boundary of this domain, three components are distributed:

$$
\begin{aligned}
& \Gamma_{0}=\{(x, 0) ; x \in[0, v=\xi(0)]\}, \\
& \Gamma_{1}=\left\{(0, t) ; t \in\left[0, t^{*}\right)\right\}, \\
& \Gamma_{g}=\left\{(x, t) ; t \in\left[0, t^{*}\right), x=\xi(t)\right\},
\end{aligned}
$$

where the initial and boundary conditions are given.

In domain $D$, we consider the heat conduction equations:

$$
\alpha \frac{\partial^{2} u(x, t)}{\partial x^{2}}=\frac{\partial u}{\partial t}(x, t),
$$

with the initial condition on boundary $\Gamma_{0}$ :

$$
u(x, 0)=\varphi(x),
$$

the Neumann condition on boundary $\Gamma_{1}$ :

$$
-k \frac{\partial u(0, t)}{\partial x}=q(t),
$$

the condition of temperature continuity and the Stefan condition on the moving interface $\Gamma_{g}$ :

$$
\begin{aligned}
u(\xi(t), t) & =u^{*}, \\
-\left.k \frac{\partial u(x, t)}{\partial x}\right|_{x=\xi(t)} & =\kappa \frac{d \xi(t)}{d t},
\end{aligned}
$$

where $\alpha$ is the thermal diffusivity, $k$ is the thermal conductivity, $\kappa$ is the latent heat of fusion per unit volume, $u^{*}$ is the phase change temperature, $x=\xi(t)$ is 
the function describing the position of the moving interface $\Gamma_{g}$, and $u, t$ and $x$ refer to temperature, time and spatial location, respectively.

The discussed inverse Stefan problem consists in finding a function to describe the temperature distribution $u(x, t)$ in domain $D$, and function $q(t)$ describing the heat flux on the boundary $\Gamma_{1}$, which will satisfy equations (2.4)-(2.8). All other functions $(\varphi(x), \xi(t))$ and parameters $\left(\alpha, k, \kappa, u^{*}\right)$, are known.

\section{Solution of the Problem}

Using the variational iteration method we are able to solve the nonlinear equation:

$$
L(u(z))+N(u(z))=f(z),
$$

where $L$ is the linear operator, $N$ is the nonlinear operator, $f$ is a known function and $u$ is a sought function. At first, we construct a correction functional:

$$
u_{n}(z)=u_{n-1}(z)+\int_{0}^{z} \lambda\left(L\left(u_{n-1}(s)\right)+N\left(\tilde{u}_{n-1}(s)\right)-f(s)\right) d s,
$$

where $\tilde{u}_{n-1}$ is a restricted variation [1, 2, 3, 4, $\lambda$ is a general Lagrange multiplier [19, 1, 2, which can be identified optimally by the variational theory [20. [1,2, 3, and $u_{0}(z)$ is an initial approximation. Next, we determine the general Lagrange multiplier and identify it as a function of $\lambda=\lambda(s)$. Finally, we obtain the iteration formula:

$$
u_{n}(z)=u_{n-1}(z)+\int_{0}^{z} \lambda(s)\left(L\left(u_{n-1}(s)\right)+N\left(u_{n-1}(s)\right)-f(s)\right) d s .
$$

The correction functional for equation (2.4) can be expressed as follows:

$$
u_{n}(x, t)=u_{n-1}(x, t)+\int_{0}^{x} \lambda\left(\frac{\partial^{2} u_{n-1}(s, t)}{\partial s^{2}}-\frac{1}{\alpha} \frac{\partial \tilde{u}_{n-1}(s, t)}{\partial t}\right) d s .
$$

From equation (3.4), the general Lagrange multiplier can be identified as follows:

$$
\lambda(s)=s-x .
$$

Hence, we obtain the following iteration formula:

$$
u_{n}(x, t)=u_{n-1}(x, t)+\int_{0}^{x}(s-x)\left(\frac{\partial^{2} u_{n-1}(s, t)}{\partial s^{2}}-\frac{1}{\alpha} \frac{\partial u_{n-1}(s, t)}{\partial t}\right) d s .
$$

Next, we select an initial approximation in the form:

$$
u_{0}(x, t)=A+B x,
$$

where $A$ and $B$ are parameters. For the determination of parameters $A$ and $B$, we will use the Neumann boundary condition (2.6) and the condition of temperature 
continuity (2.7). To this end, we require that the initial approximation $u_{0}(x, t)$ fulfils the above conditions. The boundary condition (2.6) requires:

$$
B=-\frac{1}{k} q(t),
$$

whilst the condition (2.7) leads to the result:

$$
A=u^{*}+\frac{1}{k} \xi(t) q(t) .
$$

Hence, the initial approximation has the form:

$$
u_{0}(x, t)=u^{*}+\frac{1}{k} q(t)(\xi(t)-x) .
$$

Finally, we obtain the following iteration formula:

$$
\begin{aligned}
u_{0}(x, t) & =u^{*}+\frac{1}{k} q(t)(\xi(t)-x), \\
u_{n}(x, t) & =u_{n-1}(x, t)+ \\
& +\int_{0}^{x}(s-x)\left(\frac{\partial^{2} u_{n-1}(s, t)}{\partial s^{2}}-\frac{1}{\alpha} \frac{\partial u_{n-1}(s, t)}{\partial t}\right) d s, \quad n \geq 1 .
\end{aligned}
$$

Because function $u_{n}$ (3.6) depends on an unknown function $q(t)$, we have derived this function in the form of a linear combination:

$$
q(t)=\sum_{i=1}^{m} p_{i} \psi_{i}(t),
$$

where $p_{i} \in \mathbb{R}$ and the basis functions $\psi_{i}(t)$ are a linear independence. The coefficients $p_{i}$ are selected to show a minimal deviation of function $u_{n}$ (3.6) from the initial condition (2.5) and the Stefan condition (2.8). Thus, we are looking for the minimum of the following functional:

$$
\begin{aligned}
J\left(p_{1}, \ldots, p_{m}\right)=\int_{0}^{v}\left(u_{n}(x, 0)-\varphi(x)\right)^{2} d x & \\
& +\int_{0}^{t^{*}}\left(k \frac{\partial u_{n}(\xi(t), t)}{\partial x}+\kappa \frac{d \xi(t)}{d t}\right)^{2} d t .
\end{aligned}
$$

After substituting equations (3.12) and (3.13) to functional $J$, differentiating it with respect to the coefficients $p_{i}(i=1, \ldots, m)$ and equating the obtained derivatives to zero:

$$
\frac{\partial J}{\partial p_{i}}\left(p_{1}, \ldots, p_{m}\right)=0, \quad i=1, \ldots, m,
$$

a system of linear algebraic equations is obtained. In the course of solving this system, coefficients $p_{i}$ are determined, and thereby, the approximated distributions of the heat flux $q(t)$ on boundary $\Gamma_{1}$ and temperature $u_{n}(x, t)$ in domain $D$ are obtained. 


\section{Example}

The theoretical considerations introduced in the previous sections will be illustrated with an example, where the approximate solution will be compared with an exact solution. We consider an example of the inverse Stefan problem, in which: $\alpha=0.1, k=1, \kappa=10, u^{*}=1, t^{*}=1 / 2$ and

$$
\varphi(x)=e^{-x}, \quad \xi(t)=\frac{1}{10} t .
$$

Next, an exact solution of the inverse Stefan problem will be found by means of the following functions:

$$
\begin{aligned}
u(x, t) & =e^{t / 10-x}, & & (x, t) \in D, \\
q(t) & =e^{t / 10}, & & t \in\left[0, t^{*}\right] .
\end{aligned}
$$

As basis functions we take:

$$
\psi_{i}(t)=t^{i-1}, \quad i=1, \ldots, m .
$$

In Figures 2 and 3. we present an exact and reconstructed distribution of the heat flux on the boundary $\Gamma_{1}$ for $n=1, m=5$ and for $n=2, m=2$. The left figure presents the exact (solid line) and the determined approximate position (dash line), whereas the right figure shows diagrams of the distribution of errors which occur when reconstructing the heat flux.

a)

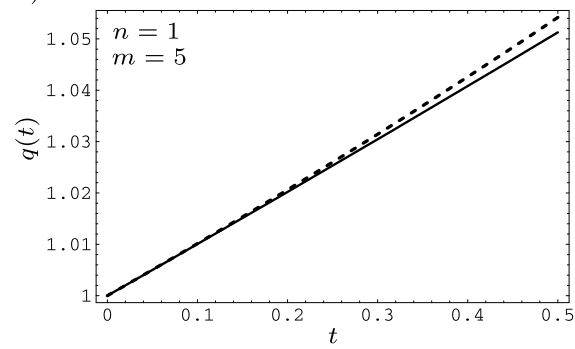

b)

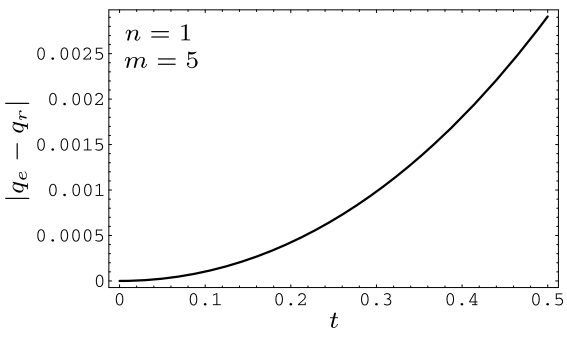

Fig. 2. Heat flux on boundary $\Gamma_{1}(a)$ and error distribution in the reconstruction of this heat flux (b) for $n=1$ and $m=5$ (solid line - exact value $q_{e}$, dash line - reconstructed value $q_{r}$ )

Figure 4 presents error distributions in the reconstruction of the phase change temperature (left figure) and error distributions in the reconstruction of the Stefan condition along the moving interface (right figure) for $n=1$ and $m=5$.

The calculations were made for an accurate moving interface position and for a position disturbed with a pseudorandom error with a size of $1 \%, 2 \%$ and $5 \%$. Table 1 presents values of the absolute error $\left(\delta_{f}\right)$ and a percentage relative error 
a)

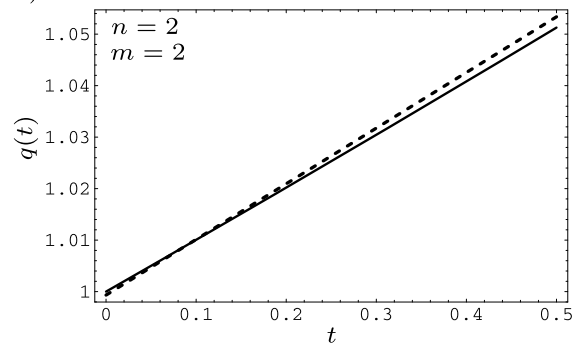

b)

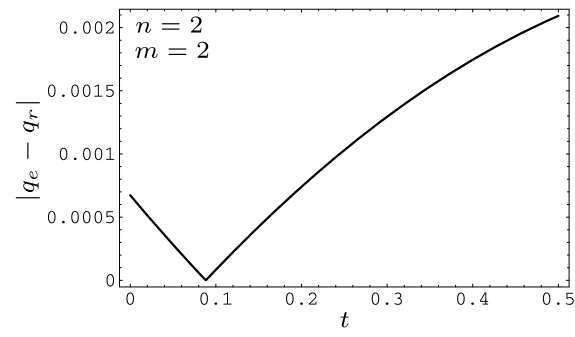

Fig. 3. Heat flux on boundary $\Gamma_{1}(a)$ and error distribution in the reconstruction of this heat flux $(b)$ for $n=2$ and $m=2$ (solid line - exact value $q_{e}$, dash line - reconstructed value $q_{r}$ )

a)

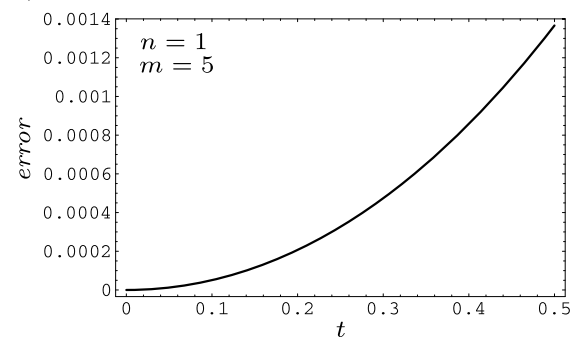

b)

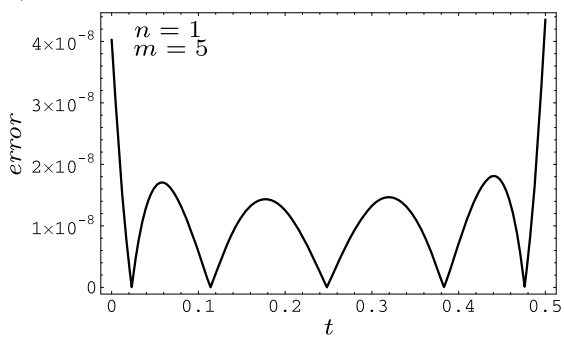

Fig. 4. Error distribution in the reconstruction of phase change temperature $(a)$ and in the reconstruction of the Stefan condition $(b)$

$\left(\Delta_{f}\right)$ with which the heat flux on the boundary $\Gamma_{1}(f=q)$ and distribution of the temperature in domain $D(f=u)$ were reconstructed for different perturbations. The values of absolute errors are calculated from formulas:

$$
\begin{aligned}
\delta_{q} & =\left(\frac{1}{t^{*}} \int_{0}^{t^{*}}\left(q_{e}(t)-q_{r}(t)\right)^{2} d t\right)^{1 / 2}, \\
\delta_{u} & =\left(\frac{1}{|D|} \iint_{D}\left(u_{e}(x, t)-u_{r}(x, t)\right)^{2} d x d t\right)^{1 / 2},
\end{aligned}
$$

where $q_{e}(t)$ is an exact value of function $q(t), q_{r}(t)$ is a reconstructed value of function $q(t), u_{e}(x, t)$ is an exact distribution of temperature in domain $D$ and $u_{r}(x, t)$ is a reconstructed distribution of temperature in this domain, and:

$$
|D|=\iint_{D} 1 d x d t
$$

However, percentage relative errors are calculated from formulas:

$$
\Delta_{q}=\delta_{q} \cdot\left(\frac{1}{t^{*}} \int_{0}^{t^{*}}\left(q_{e}(t)\right)^{2} d t\right)^{-1 / 2} \cdot 100 \%,
$$


a)

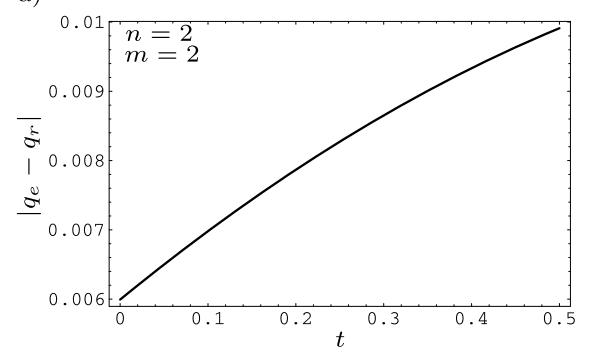

b)

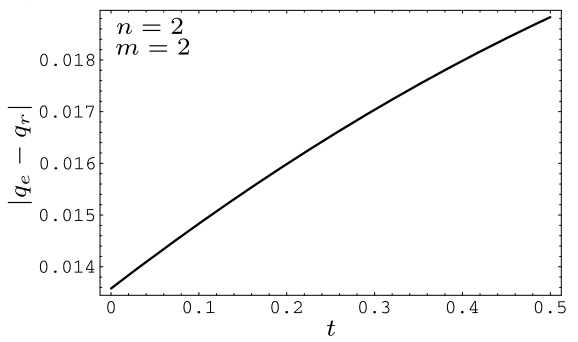

Fig. 5. Error distribution in the reconstruction of heat flux for perturbation equal to $2 \%(a)$ and $5 \%(b)\left(q_{e}\right.$ - exact value, $q_{r}$ - reconstructed value)

$$
\Delta_{u}=\delta_{u} \cdot\left(\frac{1}{|D|} \iint_{D}\left(u_{e}(x, t)\right)^{2} d x d t\right)^{-1 / 2} \cdot 100 \%
$$

As shown in the results, the presented algorithm is stable in terms of the input data errors. Each time when the input data were burdened with errors, the error of the heat flux reconstruction did not exceed the initial error.

Table 1. Values of errors in the reconstruction of heat flux and distribution of temperature ( $n=2, m=2, \delta$ - absolute error, $\Delta$ - percentage relative error)

\begin{tabular}{ccccc}
\hline Per. & $\delta_{q}$ & $\Delta_{q}$ & $\delta_{u}$ & $\Delta_{u}$ \\
\hline $0 \%$ & 0.001225 & $0.11944 \%$ & 0.000785 & $0.07721 \%$ \\
$1 \%$ & 0.002957 & $0.28830 \%$ & 0.000843 & $0.08292 \%$ \\
$2 \%$ & 0.008244 & $0.80389 \%$ & 0.001065 & $0.10473 \%$ \\
$5 \%$ & 0.016487 & $1.60768 \%$ & 0.001385 & $0.13620 \%$ \\
\hline
\end{tabular}

\section{Conclusion}

In this paper, solution of one-phase inverse Stefan problems is presented. The problem consists in a calculation of temperature distribution and of a function which describes the heat flux on the boundary, when the position of the moving interface is known. The proposed solution is based on the variational iteration method. The calculations show that this method is effective for solving the problems under consideration.

The advantage of the proposed method comparing it with classical methods consists in obtaining the heat flux and temperature distribution in the form of continuous functions, instead of a discreet form. The method applied does not require discretization of the region, like in the case of classical methods based on the finite-difference method or the finite-element method. The proposed method produces a wholly satisfactory result already in a small number of iterations, 
whereas the classical methods require a suitably dense lattice in order to achieve similar accuracy, which considerably extends the time of calculations.

\section{References}

1. He, J.-H.: Approximate analytical solution for seepage flow with fractional derivatives in porous media. Comput. Methods Appl. Mech. Engrg. 167, 57-68 (1998)

2. He, J.-H.: Approximate solution of nonlinear differential equations with convolution product nonlinearities. Comput. Methods Appl. Mech. Engrg. 167, 69-73 (1998)

3. He, J.-H.: Variational iteration method - a kind of non-linear analytical technique: some examples. Int. J. Non-Linear Mech. 34, 699-708 (1999)

4. He, J.-H.: Non-Perturbative Methods for Strongly Nonlinear Problems. Dissertation.de-Verlag im Internet GmbH, Berlin (2006)

5. He, J.-H.: Variational iteration method - Some recent results and new interpretations. J. Comput. Appl. Math. 207, 3-17 (2007)

6. Abdou, M.A., Soliman, A.A.: New applications of variational iteration method. Physica D 211, 1-8 (2005)

7. He, J.-H.: Variational iteration method for autonomous ordinary differential systems. Appl. Math. Comput. 114, 115-123 (2000)

8. He, J.-H., Liu, H.-M.: Variational approach to diffusion reaction in spherical porous catalyst. Chem. Eng. Technol. 27, 376-377 (2004)

9. He, J.-H., Wu, X.-H.: Construction of solitary solution and compacton-like solution by variational iteration method. Chaos, Solitions and Fractals 29, 108-113 (2006)

10. Momani, S., Abuasad, S.: Application of He's variational iteration method to Helmholtz equation. Chaos, Solitions and Fractals 27, 1119-1123 (2006)

11. Momani, S., Abuasad, S., Odibat, Z.: Variational iteration method for solving nonlinear boundary value problems. Appl. Math. Comput. 183, 1351-1358 (2006)

12. Słota, D.: Direct and Inverse One-Phase Stefan Problem Solved by Variational Iteration Method. Comput. Math. Appl. 54, 1139-1146 (2007)

13. Grzymkowski, R., Słota, D.: One-phase inverse Stefan problems solved by Adomian decomposition method. Comput. Math. Appl. 51, 33-40 (2006)

14. Grzymkowski, R., Słota, D.: An application of the Adomian decomposition method for inverse Stefan problem with Neumann's boundary condition. In: Sunderam, V.S., van Albada, G.D., Sloot, P.M.A., Dongarra, J. (eds.) ICCS 2005. LNCS, vol. 3516, pp. 895-898. Springer, Heidelberg (2005)

15. Zabaras, N., Yuan, K.: Dynamic programming approach to the inverse Stefan design problem. Numer. Heat Transf. B 26, 97-104 (1994)

16. Grzymkowski, R., Słota, D.: Numerical method for multi-phase inverse Stefan design problems. Arch. Metall. Mater. 51, 161-172 (2006)

17. Liu, J., Guerrier, B.: A comparative study of domain embedding methods for regularized solutions of inverse Stefan problems. Int. J. Numer. Methods Engrg. 40, 3579-3600 (1997)

18. Slodička, M., De Schepper, H.: Determination of the heat-transfer coefficient during soldification of alloys. Comput. Methods Appl. Mech. Engrg. 194, 491-498 (2005)

19. Inokuti, M., Sekine, H., Mura, T.: General use Lagrange multiplier in non-linear mathematical physics. In: Nemat-Nasser, S. (ed.) Variational Method in the Mechanics of Solids, pp. 156-162. Pergamon Press, Oxford (1978)

20. Finlayson, B.A.: The Method of Weighted Residuals and Variational Principles. Academic Press, New York (1972) 